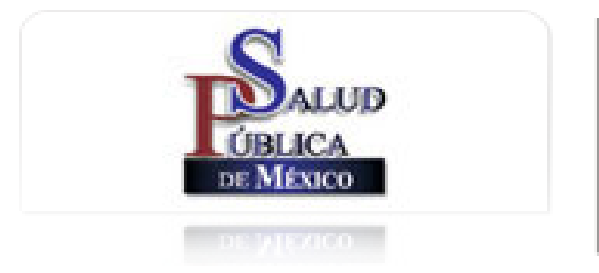

Salud Pública de México

ISSN: 0036-3634

spm@insp.mx

Instituto Nacional de Salud Pública

México

Frenk, Julio; Ruelas, Enrique; Gómez-Dantés, Octavio; López-Cervantes, Malaquías; Magaña-

Valladares, Laura; Gudiño-Cejudo, María Rosa

Modernización académica de la Escuela de Salud Pública de México: 1983-1995

Salud Pública de México, vol. 55, núm. 5, septiembre-octubre, 2013, pp. 528-535

Instituto Nacional de Salud Pública

Cuernavaca, México

Disponible en: http://www.redalyc.org/articulo.oa?id=10628334011

Cómo citar el artículo

- Número completo

- Más información del artículo

- Página de la revista en redalyc.org

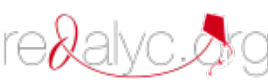

Sistema de Información Científica

Red de Revistas Científicas de América Latina, el Caribe, España y Portugal

Proyecto académico sin fines de lucro, desarrollado bajo la iniciativa de acceso abierto 


\title{
Modernización académica \\ de la Escuela de Salud Pública de México: 1983-1995
}

\author{
Julio Frenk, MC, PhD,(1) Enrique Ruelas, MC, MAP, MASS, ${ }^{(2,3)}$ Octavio Gómez-Dantés, MC, MSP, ${ }^{(4)}$ \\ Malaquías López-Cervantes, MC, PhD, ${ }^{(5)}$ Laura Magaña-Valladares, D en E, ${ }^{(6)}$ María Rosa Gudiño-Cejudo, D en H. ${ }^{(6)}$
}

\section{Frenk J, Ruelas E, Gómez-Dantés O, López-Cervantes M, Magaña-Valladares L, Gudiño-Cejudo MR. Modernización académica de la Escuela de Salud Pública de México: 1983-1995. Salud Publica Mex 2013;55:528-535.}

\begin{abstract}
Resumen
Este artículo discute los esfuerzos por modernizar la Escuela de Salud Pública de México (ESPM). En la primera parte se abordan las condiciones en las que operaba la Escuela a principios de la década de los ochenta. En la segunda parte se describen los cambios introducidos entre 1983 y 1986, hasta antes de la incorporación de la ESPM al Instituto Nacional de Salud Pública (INSP). En la tercera parte se revisa, sobre todo, la renovación conceptual que dio sustento a los nuevos programas docentes que se implantaron a finales de los años ochenta y las iniciativas implantadas entre 1987 y 1995 . En la cuarta parte se discute la construcción de la nueva sede del INSP y la mudanza a Cuernavaca. Este ensayo busca comunicar la importancia de las transformaciones por las que atravesó la ESPM y que la convirtieron en una institución verdaderamente académica que utiliza la investigación como motor de la formación de los recursos humanos en salud pública.
\end{abstract}

Palabras clave: Escuela de Salud Pública de México; educación en salud pública
Frenk J, Ruelas E, Gómez-Dantés O, López-Cervantes M, Magaña-Valladares L, Gudiño-Cejudo MR.

Academic modernization of the Mexican School

of Public Health: 1983-1995.

Salud Publica Mex 2013;55:528-535.

\section{Abstract}

In this paper we discuss the recent efforts to modernize the Mexican School of Public Health (ESPM). In the first part we analyze the conditions in which the ESPM operated at the beginning of the 1980s. In part two we describe the changes introduced in the ESPM between 1983 and 1986, up until its incorporation into the National Institute of Public Health (INSP). In part three we discuss the conceptual renovation which built the platform for the new academic programs introduced by the School between 1987 and 1995. In the fourth part we discuss the construction of the new facilities of INSP and the process of moving to the city of Cuernavaca. The main message of this paper is that the transformations witnessed by the ESPM in the final decades of the past century turned it into a true academic institution which uses research as the motor for training of human resources in public health.

Kew words: Mexican School of Public Health; training in public health

(I) Facultad de Salud Pública, Universidad Harvard. Boston, MA, EUA.

(2) Academia Nacional de Medicina. México, DF, México.

(3) Institute for Healthcare Improvement. Cambridge, MA, EUA.

(4) Centro de Investigación en Sistemas de Salud, Instituto Nacional de Salud Pública. Cuernavaca, Morelos, México.

(5) Unidad de Proyectos Especiales de Investigación Sociomédica, Facultad de Medicina, Universidad Nacional Autónoma de México. México, DF, México

(6) Secretaría Académica, Instituto Nacional de Salud Pública. Cuernavaca, Morelos, México.

\footnotetext{
Fecha de recibido: 8 de marzo de 2013 • Fecha de aceptado: 6 de junio de 2013

Autor de correspondencia: Dr. Octavio Gómez-Dantés. Genovevo de la O, 103 A, col. Santa María Ahuacatitlán. 62100 Cuernavaca, Morelos, México.

Correo electrónico: ocogomez@yahoo.com
} 
$E^{n}$ el último cuarto del siglo $\mathrm{XX}$ se produjeron en México cambios que modificaron de manera radical el perfil epidemiológico del país y el rostro de las instituciones de salud. El acelerado proceso de envejecimiento de la población mexicana empezaba a reflejarse en un creciente predominio de los padecimientos no transmisibles, como las enfermedades cardiovasculares, el cáncer y la diabetes. Ya en 1980, dichos padecimientos fueron responsables de la mitad de las defunciones totales, mientras que las infecciones y los padecimientos relacionados con la reproducción y la nutrición concentraron un tercio de los decesos. ${ }^{1}$

Este nuevo panorama epidemiológico demandaba cambios en la respuesta social organizada que no se hicieron esperar. De hecho, en la década de los ochenta se produjo lo que se podría considerar la segunda generación de reformas del sistema mexicano de salud. ${ }^{2}$ En 1983 el derecho a la protección de la salud se incorporó al artículo $4^{\circ}$ constitucional. ${ }^{3}$ Un año más tarde se promulgó la ley reglamentaria de este artículo, la Ley General de Salud, que sustituyó a un código sanitario caduco. ${ }^{4}$ En 1985 la Secretaría de Salubridad y Asistencia pasó a llamarse Secretaría de Salud y puso en marcha la descentralización de los servicios de salud para la población no asegurada, lo cual dio origen a los Servicios Estatales de Salud. 5,6

Los cambios en las condiciones e instituciones de salud exigían una nueva generación de profesionales de la salud pública capaces de enfrentar los retos de la transición epidemiológica haciendo un uso equilibrado y efectivo de los conceptos y métodos de la estadística y la epidemiología modernas, del naciente campo de los sistemas de salud y de las novedosas herramientas de la informática. La Escuela de Salud Pública de México (ESPM), fundada en 1922, parecía el nicho ideal para formar a los cuadros que requería el revolucionado sector salud mexicano. Sin embargo, con planes, programas y técnicas de enseñanza anticuados, y habiendo perdido el vínculo con la investigación como consecuencia de la separación organizacional con el Instituto de Salubridad y Enfermedades Tropicales (ISET) en 1959, y la separación física con el cambio de sede en 1970, la ESPM demandaba una renovación que la situara a la altura de los tiempos. Este artículo cuenta precisamente esa historia: la de los esfuerzos por modernizar y fortalecer académicamente a la escuela de salud pública más antigua de América Latina. ${ }^{*}, 8$ Estos esfuerzos se pueden agrupar en tres etapas que se resumen en el cuadro I y que se describen extensamente a lo largo de este ensayo: de recambio, modernización y consolidación.

En la primera parte de este artículo se discuten el contexto y las condiciones en las que operaba la ESPM a principios de la década de los años ochenta. En la segunda parte se describen los cambios introducidos entre 1983 y 1986, hasta antes de la incorporación de la ESPM al Instituto Nacional de Salud Pública (INSP). La tercera parte se dedica a revisar sobre todo la renovación conceptual que dio sustento a los nuevos programas docentes que se implantaron en la escuela a finales de los años ochenta y hacer un recuento de las principales iniciativas implantadas en la ESPM entre 1987 y 1995, año éste último en que se integra plenamente al INSP, dando pie al establecimiento de la Secretaría Académica del Instituto. En la cuarta parte se discute el proceso de construcción de la nueva sede del INSP en la ciudad de Cuernavaca. El principal mensaje de este ensayo es que las transformaciones por las que atravesó la ESPM la convirtieron en una institución verdaderamente académica que utiliza la investigación como motor de la formación de los recursos humanos en salud pública y que ha contribuido a responder de manera racional a los retos que enfrenta el sistema mexicano de salud en el siglo XXI.

\footnotetext{
* Ha habido una larga discusión sobre si la ESPM, fundada en 1922, es la escuela de salud pública más antigua de América Latina o si lo es la Facultad de Salud Pública de la Universidad de Sao Paulo. En la página electrónica de esta facultad se señala que su institución precursora se creó en 1918.7 Sin embargo, lo que se fundó ese año dentro de la Facultad de Medicina de Sao Paulo (la cual se integró a la Universidad de Sao Paulo en los años treinta), con el apoyo de la Fundación Rockefeller, fue el Laboratorio de Higiene, que no tenía funciones de formación de recursos humanos. En 1923 dicho laboratorio se desvinculó de la Facultad de Medicina para crear el Instituto de Higiene. ${ }^{8}$ Fue hasta que se creó este instituto que se empezaron a formar recursos humanos en salud pública en Brasil, un año después de fundada la Escuela de Salud Pública de México.
}

\section{Cuadro I}

\section{Etapas de la modernización de la Escuela de Salud Pública de México, 1970-1995}

\begin{tabular}{lcl} 
Periodo & Denominación & \multicolumn{1}{c}{ Sucesos relevantes } \\
$1970-1982$ & Estancamiento & Gradual obsolescencia de los planes y programas docentes \\
\hline $1983-1986$ & Recambio & $\begin{array}{l}\text { Inicio del abandono de la formación técnica, renovación del programa de maestrías en ciencias y recuperación } \\
\text { de las actividades de investigación }\end{array}$ \\
\hline $1987-1990$ & Modernización & $\begin{array}{l}\text { Diseño de una base conceptual para el desarrollo de las actividades docentes, consolidación de las maestrías } \\
\text { en ciencias y fortalecimiento de la vinculación docencia/investigación }\end{array}$ \\
\hline $1990-1995$ & Consolidación & Diversificación de las maestrías en ciencias, y diseño y puesta en operación del doctorado en salud pública
\end{tabular}




\section{Creación, desarrollo y estancamiento de la ESPM}

Desde principios de los años veinte del siglo pasado, la Escuela de Salubridad formó a una buena parte de los cuadros que demandaba el sistema de salud: oficiales médicos y enfermeras de salubridad, especialistas en servicios sanitarios, técnicos laboratoristas, técnicos en saneamiento, técnicos inspectores y, eventualmente, maestros en salud pública.

La fusión, en 1943, de la Secretaría de Asistencia Pública con el Departamento de Salubridad para dar origen a la Secretaría de Salubridad y Asistencia le dio un nuevo impulso. Ubicada dentro de la Subsecretaría de Salubridad, a la denominada en 1938 Escuela de Salubridad e Higiene se le asignaron como tareas la educación y el adiestramiento del personal sanitario; la orientación y unificación de la enseñanza de la higiene y la medicina preventiva en la República, y la coordinación de las actividades educativas sobre la higiene con otras instituciones oficiales y privadas. ${ }^{9,10}$

La formación de recursos humanos en salud pública se fortaleció al vinculársele con la investigación cuando en 1954 se fusionaron la Escuela de Salubridad e Higiene y el ISET para dar origen a la Escuela e Instituto de Salubridad y Enfermedades Tropicales. Este enlace duró solo cuatro años, ya que en 1958 se separaron formalmente, aunque siguieron compartiendo una misma sede hasta 1970. ${ }^{9}$ No obstante esto, buena parte del personal del ISET siguió dando clases en la Escuela. De hecho, la plantilla de profesores en este último año estaba encabezado por un grupo de primerísimo nivel: Gerardo Varela en bacteriología, Manuel Martínez Báez en parasitología, Miguel E. Bustamante en epidemiología, Gonzalo Aguirre Beltrán y Ricardo Pozas Arciniégas en antropología, Joaquín Cravioto y Adolfo Chávez en nutrición, Humberto Romero Álvarez en saneamiento ambiental y Pedro Daniel Martínez en administración. ${ }^{11}$

El fortalecimiento de la capacitación en salud pública contribuyó al buen desempeño de las campañas y programas de control de enfermedades que se implementaron en los cincuenta (Campaña de Erradicación de la Viruela, Campaña Nacional para la Erradicación del Paludismo, Programa de Lucha contra la Tuberculosis), a la creación de los programas preventivos del IMSS y de salud pública en los Servicios Coordinados de Salud Pública, y a la consolidación de las cátedras de medicina preventiva y salud pública en las escuelas y facultades de medicina de todo el país. ${ }^{11}$

Los años sesenta fueron testigo de un desarrollo explosivo de la infraestructura hospitalaria pública para la salud que influyó en los programas de la denominada, desde 1959, Escuela de Salud Pública (ESP). Entre 1959 y 1964 el número de hospitales del Instituto Mexicano del Seguro Social (IMSS), creado en 1943, casi se duplicó; el recién creado Instituto de Seguridad y Servicios Sociales de los Trabajadores del Estado (ISSSTE) construyó 33 unidades hospitalarias, y la Secretaría de Salubridad y Asistencia amplió en 500 unidades su infraestructura nosocomial. ${ }^{12,13}$ Una de las respuestas de la Escuela a este asombroso crecimiento fue la ampliación del ámbito de docencia de la Maestría en Salud Pública mediante la creación de la Maestría en Salud Pública y Administración Médica.

Por estas fechas, la influencia de la ESP empezó a extenderse a España, América Latina y el Caribe al convertirse en el principal centro formador de recursos humanos en salud pública de América Central y los países andinos, en particular Colombia, Ecuador, Perú y Venezuela. ${ }^{9}$ De hecho, desde 1953, las escuelas de salud pública de México, Brasil y Chile estaban registradas ante la Oficina Sanitaria Panamericana como las únicas con estudiantes extranjeros, principalmente latinoamericanos. ${ }^{14}$

En 1970 la ESP mudó su sede al sur de la Ciudad de México, a un edificio originalmente diseñado como hospital que no ofrecía condiciones óptimas para el desarrollo de sus funciones. Este cambio marcó el inicio de una etapa de paulatino estancamiento, que se reflejó, a juicio de varios de los testigos de ese proceso que fueron entrevistados para la elaboración de este artículo, en un envejecimiento de los programas docentes, un peso creciente de la formación de personal técnico, una persistente endogamia y una cultura institucional autoritaria.

Los programas docentes que se impartían en la ESP en los años setenta seguían basándose en conocimientos y textos de los años cincuenta y no incorporaban los extraordinarios avances metodológicos que se produjeron en el campo de la epidemiología en los setenta; la estadística que se enseñaba era meramente descriptiva, y poca atención se prestaba a las innovaciones que se estaban produciendo en las ciencias de la administración. A eso habría que agregar el poco acceso que se tenía a las revistas líderes en salud pública, epidemiología, servicios de salud y salud ambiental, entre otras.

Los llamados posgrados profesionalizantes (Maestría en Salud Púbica y Maestría en Administración de Hospitales) concentraban sólo una parte de la carga docente crecientemente orientada a la impartición de cursos técnicos dirigidos a enfermeras, técnicos de laboratorio $\mathrm{y}$ trabajadores comunitarios.

El carácter endogámico de la ESP fue resultado de su aislamiento institucional y disciplinario. La mayoría de los docentes eran egresados de la propia escuela, los lazos con otras instituciones nacionales de formación de recursos humanos para la salud eran escasos y era proverbial la pérdida de vínculos con los principales centros internacionales de investigación y formación de recursos humanos en salud pública, como la Escuela de Higiene y Salud Pública de la Universidad Johns Hopkins y la Escuela de Salud Pública de la Universidad Harvard, instituciones estas últimas que habían formado a los primeros salud pública de méxico / vol. 55, no. 5, septiembre-octubre de 2013 
docentes que tuvo la escuela en los años veinte (Salvador Bermúdez, Miguel E. Bustamante, Gabriel Garzón Cossa, Ricardo Granillo, Maximiliano Ruiz Castañeda y Gerardo Varela). ${ }^{10}$ La ESP, además, se había negado a incorporar a los programas a maestros y aspirantes que no se hubieran formado en el campo de las ciencias de la salud, con la sola excepción de los ingenieros, los cuales jugaban un papel crucial en las tareas de saneamiento. A esto habría que agregar el poco contacto que los docentes y alumnos tenían con las actividades de investigación desde que la ESP se separó del ISET.

El corolario de este proceso de estancamiento y aislamiento fue el desarrollo de una cultura más burocrática que académica, inserta como estaba la ESP en la estructura vertical de una secretaría de Estado que era parte, a su vez, de un régimen autoritario. Lejos de estimularse la independencia inquisitiva, esencial para el quehacer académico, se penalizaba la crítica.

Otro factor que dificultó la adaptación de la ESP a los cambios que se produjeron en los años setenta y principios de los ochenta en el país, en general, y en las instituciones de salud y educativas, en particular, fue su inestabilidad administrativa: en un sexenio dependía de la Oficialía Mayor y en el siguiente, de la Subsecretaría de Salubridad. Algo que facilitó su eventual modernización fue su desconcentración administrativa, que se produjo en 1982, un año después de que adquiriese la denominación de ESPM. ${ }^{15,16}$

\section{Inicio de la modernización de la ESPM}

Los cambios en el sector salud de principios de los ochenta que se mencionan en la introducción de este ensayo crearon las condiciones para empezar a superar la larga crisis a la que se enfrentaba la ESPM. De hecho, una de las diez estrategias del llamado "cambio estructural en la salud" implantado a principios de los años ochenta era precisamente la renovación de la investigación y la formación de los recursos humanos para la salud, las cuales se definieron como "instrumentos del cambio". 17,18

Los antecedentes académicos de los principales funcionarios de la nueva administración de la Secretaría de Salubridad y Asistencia contribuyeron también a modificar la inercia con la que se venían formando los profesionales de la salud pública en México. Algunos de estos funcionarios, de hecho, habían estado involucrados en la renovación de la enseñanza de la salud pública en la Facultad de Medicina de la Universidad Nacional Autónoma de México (UNAM), y en los años setenta habían tenido la oportunidad de visitar los principales centros de formación de recursos humanos en salud pública del mundo anglosajón. Algunos de ellos habían organizado un histórico seminario internacional realizado en Querétaro, México en 1978 para impulsar la enseñanza y la investigación en servicios de salud. ${ }^{19}$
En la segunda mitad de los setenta, un numeroso grupo de jóvenes médicos, egresados la mayoría de ellos de la Facultad de Medicina de la UNAM, decidieron especializarse en salud pública y llevaron a cabo estudios de maestría y doctorado en las escuelas de salud pública de Harvard, Johns Hopkins, Yale y Michigan, en la División de Salud Comunitaria de la Facultad de Medicina de la Universidad de Toronto, y en la Escuela de Higiene y Medicina Tropical de Londres. Este grupo ayudó a renovar la docencia en la ESPM a principios de los ochenta y a su alrededor se empezaría a generar la masa crítica de investigadores que daría origen al INSP.

Según José Rodríguez Domínguez, a principios de los años ochenta, las condiciones en el país y en el sector salud "estaban puestas... para hacer un cambio [en la ESPM] que históricamente significara una actualización del proyecto original de 1922". ${ }^{20}$ A finales de 1982 cambió la administración de la ESPM y de inmediato se hizo explícito el propósito de renovarla académicamente, modernizando los programas docentes, retomando su vínculo con la investigación y orientándola sobre todo a la formación de posgrado.

Muy pronto se empezó a planear e implantar una revisión evaluativa del desempeño de la ESPM a partir de un "libro blanco" que ya hablaba de la necesidad de modernizar la institución. Una de las ideas que surgió de este proceso fue la necesidad de fortalecer los cursos metodológicos y re-introducir la investigación para crear maestrías en ciencias y eventualmente programas de doctorado. En esta evaluación participó la Organización Panamericana de la Salud, que envió una delegación encabezada por los doctores Abraham Horwitz y José Roberto Ferreira. Esta delegación generó un conjunto de recomendaciones para modernizar la ESPM. Del informe final destaca la siguiente observación:

Interpretamos la situación como una crisis de identidad de la escuela en función del desajuste entre los niveles de conocimiento que maneja y el escenario mucho más amplio en que actúa la salud pública. La escuela, aún considerada como de posgrado, desarrolla programas de educación superior especializada y de capacitación a nivel intermedio descuidando la excelencia técnica de su programación por la limitada participación en actividades de investigación científica. ${ }^{21}$

Una de las primeras medidas tomadas por la nueva administración de la ESPM fue transferir un número importante de programas de capacitación técnica al Colegio Nacional de Educación Profesional Técnica (Conalep), institución educativa de nivel medio superior creado en 1978. Esto permitió concentrar los recursos de la escuela en los programas de posgrado.

Al mismo tiempo se empezó a renovar y fortalecer el primer posgrado académico con el que había contado 
la ESPM: la Maestría en Ciencias en Epidemiología, que se había creado a finales de los años setenta. Se rediseñó en estrecha colaboración con el grupo que esos mismos años estaba rediseñando la Maestría en Ciencias Sociomédicas con Concentración en Epidemiología de la Facultad de Medicina de la UNAM, la cual se convertiría en Maestría en Epidemiología. ${ }^{22}$ Un claro indicador del éxito de la Maestría en Ciencias en Epidemiología de la ESPM es que siete de los egresados de las tres primeras generaciones del nuevo programa completaron estudios de doctorado en diversas escuelas de salud pública, algunos en el INSP y otros en universidades extranjeras. Por esas fechas también se creó la Residencia en Epidemiología, orientada sobre todo a formar cuadros para el Sistema Nacional de Vigilancia Epidemiológica, un programa conjunto de la Secretaría de Salud de México y el Centro para el Control de Enfermedades (CDC por sus siglas en inglés) de Estados Unidos. ${ }^{23}$

Una medida que parece poco relevante, pero que habla del atraso con el que había operado la ESPM, fue la incorporación del uso de las computadoras en los cursos regulares y los trabajos de tesis. Esto dio inicio con la organización de diversas actividades de capacitación en cómputo utilizando las terminales de la única computadora con la que en ese entonces contaba la Secretaría de Salud, ubicada en el área de recursos humanos. En 1984 la ESPM compró las primeras computadoras y para 1986 ya contaba con más de cincuenta. ${ }^{20}$

Aunque en general hubo poca resistencia de los docentes a los cambios implementados por la nueva administración, vale la pena mencionar que el titular de los cursos de estadística de la ESPM renunció al enterarse que los estudiantes empezarían a utilizar computadoras y un software estadístico, SPSS. Argumentó que no estaba de acuerdo con la utilización de máquinas que iban a sustituir la capacidad y la inteligencia de los individuos, quienes no iban a entender qué era y para qué era la estadística.

Un hecho que contribuyó de manera fundamental a vincular la enseñanza con la investigación en salud pública en la ESPM fue la creación del Centro de Investigaciones en Salud Pública (CISP) el 20 de agosto de 1984 con el propósito de producir evidencias científicas para racionalizar la toma de decisiones. ${ }^{24}$ La ESPM de inmediato se ligó estrechamente con este nuevo centro con el propósito explícito de hacer de la investigación el motor de la docencia. Los investigadores del CISP, además, empezaron a participar crecientemente en las actividades de enseñanza de la escuela.

\section{Consolidación académica de la ESPM}

Un acontecimiento que fortaleció los vínculos de la ESPM y el CISP, y que de alguna manera anunció la integración de estas dos instituciones para dar origen al INSP, fue la estrecha colaboración en el proceso de reconstrucción de los servicios de salud en el Valle de México después de los sismos de $1985 .{ }^{25}$ En diversos documentos que tuvieron origen en el CISP se afirmaba que esta reconstrucción ofrecía la oportunidad de reorganizar los servicios bajo un nuevo modelo que privilegiara la atención primaria y descentralizara la atención hospitalaria. ${ }^{26,27}$

La participación de las dos instituciones en el proceso de reconstrucción lo facilitó la existencia de un programa conjunto de la ESPM y el CISP que se había creado en 1985 con el apoyo de la Fundación Kellogg: el Programa de Educación Avanzada en Organización y Dirección de Servicios de Salud (Proasa, México). En colaboración con el CISP y el Centro de Capacitación y Desarrollo de la Secretaría de Salud, la ESPM creó, en 1986, el Programa de Formación de Directivos para los Nuevos Hospitales de la Reconstrucción dirigido a la formación de once distintos tipos de directivos para cada uno de los nuevos hospitales. ${ }^{28}$ El CISP y la ESPM se encargaron del diseño de los contenidos conceptuales del programa, mientras que el Centro de Capacitación y Desarrollo se responsabilizó del diseño de los contenidos técnicos. Un año después este programa se complementó con una iniciativa de formación de recursos humanos en salud pública, el Programa Continuado de Formación de Directivos dirigido a directores de hospitales en funciones, que ofrecía, durante un año, sesiones mensuales de actualización en administración de servicios de salud.

Cabe destacar que estas iniciativas docentes se acompañaron de una estrategia de investigación que también implementaron de manera conjunta la ESPM y el CISP denominado "Análisis de problemas médicos directivos en diferentes organizaciones hospitalarias". Este proyecto permitió definir una tipología de problemas, las variaciones que en la percepción de éstos existe en los diferentes niveles jerárquicos y la influencia que las distintas estructuras organizacionales ejercen en los diferentes tipos de retos. Este proyecto generó varias publicaciones cuyo objetivo era dar a conocer las lecciones del programa de formación de directivos para eventualmente reproducirlo en otros países de América Latina. ${ }^{29-32}$

Estos procesos de colaboración a principios de 1987 dieron un salto cuántico con la creación del INSP mediante la integración funcional del CISP, la ESPM y el Centro de Investigación sobre Enfermedades Infecciosas (CISEI). El INSP generó la infraestructura básica para, en palabras del entonces secretario de Salud, "igualar en el área de la salud pública la excelencia y prestigio internacional logrado por la investigación biomédica mexicana". ${ }^{18}$

La nueva institución nació al abrigo de una novedosa propuesta conceptual en la que se identifican de manera muy precisa las tareas que habría de desarrollar la ESPM en materia de formación de recursos humanos 
para la salud. En un documento que se presentó en junio de 1987 en el simposio internacional "La salud pública hacia el siglo XXI: estrategias para la educación superior y la investigación", se define a la salud pública como la aplicación de las ciencias biológicas, sociales y administrativas en el estudio de los fenómenos de salud en poblaciones humanas, y se le identifica como un campo de conocimiento y como un ámbito para la acción. ${ }^{33}$ Ahí mismo se señala que como campo de conocimiento la salud pública requiere de una función de investigación para la producción y otra de enseñanza para la reproducción de dicho conocimiento.

Para los propósitos de este ensayo, lo más relevante del documento arriba citado, que por cierto se nutre de un artículo que estableció las bases conceptuales para la investigación en salud pública en el CISP, ${ }^{34}$ es la discusión sobre los diversos tipos de formación de recursos humanos para la salud pública y el nicho que ocuparía la ESPM dentro del INSP. Reconocía dos formas básicas de desarrollo de recursos humanos en este campo: la educación superior y la capacitación para el trabajo. La educación superior a la vez comprende la formación de recursos humanos y la educación continua. Finalmente, la formación de recursos humanos incluye la especialización y la educación a través de programas de maestría y doctorado. El artículo señala que la mayor parte de las escuelas de salud pública de América Latina, como sugiere el informe de Horwitz y Ferreira antes citado, han sufrido las consecuencias de la falta de diferenciación entre capacitación para el trabajo y educación superior. La alternativa era sustituir las pocas instituciones centralizadoras por una red de organizaciones con diferenciación funcional:

Un sistema así tendría en su base a un conjunto descentralizado de unidades locales de capacitación encargadas de satisfacer los requerimientos de corto plazo de los organismos de salud. El segundo nivel estaría representado por un número limitado de escuelas regionales de salud pública que servirían a varios estados impartiendo primordialmente programas de especialización y maestría en salud pública. En este marco, la misión de dar una integración nacional al sistema correría a cargo de un instituto que, al concentrarse en programas de maestría en ciencias, doctorado y educación continua, prepararía a los docentes e investigadores para los otros niveles, generando un verdadero flujo de "referencia" y "contrarreferencia" educativas.

Ya integrada al INSP, la ESPM diseñó un Plan de Desarrollo de la Escuela de Salud Pública de México 1987-1988, el cual estableció que el objetivo de esta institución era formar y desarrollar recursos humanos de alto nivel académico mediante programas de especialización, maestría y doctorado. Los programas de especialización se orientarían a la formación de recursos humanos dedicados principalmente a la práctica profesional, mientras que los programas de maestría y doctorado enfatizarían la formación para la docencia y la investigación en salud pública. Esto obligó también a tramitar el cambio de registro de la ESPM ante la Secretaría de Educación Pública de institución de educación media y normal a institución de educación superior.

Con el Plan de Desarrollo surgieron nuevos programas que buscaban atender retos apremiantes. En enero de 1987 arrancó un nuevo programa de maestría financiado por la Fundación Kellogg: la Maestría en Organización y Dirección de Sistemas de Atención a la Salud, que más tarde se denominó Maestría en Ciencias en Sistemas de Salud. En septiembre de ese mismo año dio inicio la Especialidad en Salud Ambiental en colaboración con el Centro Panamericano de Ecología Humana y Salud. Más tarde se echó andar la Maestría en Salud Pública en Salud Mental en colaboración con el Instituto Mexicano de Psiquiatría. Cabe señalar que el diseño curricular de los nuevos programas y el rediseño de los existentes se llevó a cabo también con el apoyo de expertos del Centro Universitario de Tecnología Educacional para la Salud (CEUTES) de la UNAM.

Los nuevos programas académicos enriquecieron la oferta de la ESPM que seguía incluyendo a la Maestría en Salud Pública (MSP), la Especialidad en Administración de Hospitales, la Especialidad de Enfermería en Salud Pública y un renovado Programa de Educación Continua. La MSP, uno de los programas más antiguos de la escuela, pasó también por un proceso de renovación a partir de un ejercicio de planeación prospectiva en el que participaron 240 expertos en el área promovido por la ESPM, la Sociedad Mexicana de Salud Pública y la Asociación Mexicana de Educación en Salud Pública, con el apoyo de la Fundación Barros Sierra. ${ }^{35}$ Años más tarde, se creó la Maestría en Salud Pública semipresencial que fortaleció los vínculos de la ESPM con los servicios de salud al capacitar en salud pública a un número importante de directivos estatales y jurisdiccionales de dichos servicios. Además, cabe destacar que casi todos estos programas se incorporaron al padrón de excelencia del Consejo Nacional de Ciencia y Tecnología.

Una de las medidas que más contribuyó a democratizar la ESPM fue la creación de la Comisión Académica interna, en las que estaban representados tanto el personal académico como los estudiantes. Esta comisión, entre otras cosas, diseñó y puso en operación criterios explícitos, auditables y estrictamente académicos para la selección de alumnos, medida que representó un avance enorme en relación con los criterios discrecionales que antes se utilizaban en este proceso de selección.

Un claro signo del éxito de la modernización y la consolidación académica de la ESPM fue el diseño y 
puesto en marcha, en 1991, del Doctorado en Ciencias en Salud Pública en las especialidades de epidemiología y sistemas de salud, que representó el primer programa en su género en América Latina.

En la primera mitad de los años noventa se incorporaron nuevos programas docentes a la ESPM, dentro de los que destacan la Maestría en Ciencias en Salud Reproductiva en 1991 y las Maestrías en Ciencias en Higiene Ocupacional y en Economía de la Salud en 1995; esta última es la primera en su género en el mundo de habla hispana.

\section{La nueva sede}

Un componente complementario al proceso de modernización académica de la ESPM que se asocia con su incorporación al INSP fue la construcción de la sede definitiva de este instituto, que albergaría en un mismo espacio físico a las tres unidades académicas que inicialmente lo conformaron. La idea básica original era la construcción de una obra que fuera la expresión física de "la nueva salud pública". Se acordó construirla en la ciudad de Cuernavaca, ubicada a $80 \mathrm{~km}$ al sur de la Ciudad de México, para así contribuir al proceso de descentralización del gobierno federal.

Lo primero que se hizo fue buscar un terreno. Muy pronto se identificó un amplio espacio al norte de la ciudad de Cuernavaca. Era un terreno con pronunciados y atractivos desniveles. El proyecto se estructuró en torno a una explanada o plaza en la que convergerían los tres edificios: laboratorios de investigación, bioterio y un espléndido edificio central para albergar las aulas, los auditorios, los laboratorios de enseñanza, los cubículos de los investigadores, las áreas administrativas y las oficinas de los directivos de todas las unidades académicas. El edificio central se diseñó sobre la base de una concepción del trabajo académico, creando un amplio atrio central y diversos espacios que alentarían la comunicación entre los miembros de la comunidad académica. En el borde de las escalinatas de acceso a la explanada se ubicó una escultura "pivote" diseñada exprofeso por Sebastián: "La escalera cósmica". El edificio se inauguró el 24 de enero de 1991.

La incorporación de la ESPM al INSP tuvo, además de un componente organizacional y otro arquitectónico, un componente humano que no debe subestimarse: la mudanza de parte del personal de la ESPM y del CISP a la ciudad de Cuernavaca, y la movilización diaria de otra parte de la comunidad que seguía residiendo en la capital del país. Convencer a parte del personal de mudarse a Cuernavaca no fue difícil: había un alto nivel de motivación pues muchos de los investigadores residentes en la Ciudad de México todavía padecían los estragos de los sismos de 1985 y se consiguió un razonable paquete de créditos hipotecarios. El traslado del personal que seguía residiendo en el Distrito Federal se resolvió en parte con la compra de un autobús que hacía viajes diarios del sur del Distrito Federal a Cuernavaca temprano por la mañana y de regreso por las tardes. El balance de este proceso, en general, fue favorable, pero no faltaron algunas experiencias desagradables que lo complicaron, dentro de las que destaca un fraude inmobiliario del que fueron víctimas alrededor de 20 investigadores.

\section{Conclusión}

La modernización académica que se produjo en la ESPM a finales del siglo pasado y que la llevó a una etapa de madurez fue posible gracias a la conjunción de distintos sucesos: la transición epidemiológica por la que atravesaba el país, la llegada a la entonces Secretaría de Salubridad y Asistencia de un nutrido grupo de funcionarios con antecedentes académicos que encabezaron un ambicioso proceso de reforma del sector salud y el surgimiento de una generación de jóvenes médicos mexicanos que hicieron estudios de posgrado en las mejores escuelas de salud pública del mundo y que regresaron al país justo en esta etapa de cambio.

La modernización de la ESPM incluyó la renovación de los contenidos de las maestrías tradicionales, tanto en el ámbito conceptual como metodológico; la renovación y ampliación del programa de maestrías en ciencias; el desarrollo de la investigación tanto en epidemiología como en sistemas de salud; la formación en la utilización de las herramientas informáticas y su uso en las tareas de investigación, y el fortalecimiento de los lazos con otras instituciones académicas nacionales e internacionales, así como con los principales organismos multilaterales y organizaciones filantrópicas dedicadas a la salud. Posiblemente el signo más claro de madurez académica de la ESPM fue la inauguración del programa de doctorado en 1991.

Pero no todo fue miel sobre hojuelas. En su renovación, la ESPM tuvo que luchar contra inercias institucionales y prácticas burocráticas propias de un régimen que había permanecido en el poder más de 60 años. Algunas de estas prácticas no habrían de eliminarse por completo sino hasta bien entrado el siglo XXI, cuando el proceso de democratización por el que pasaron nuestras instituciones estuvo bien enraizado.

No obstante los innegables avances, es importante reconocer que, ya modernizada, la ESPM ha tenido que enfrentar retos diversos dentro de los que destacan la atomización de sus programas de maestría; la especificación incompleta de las competencias que los estudiantes deben desarrollar; el insuficiente aprovechamiento de las ventajas comparativas, dentro de las que se incluye su cercanía con la formulación de las políticas públicas, y la lucha continua contra las tradiciones burocráticas 
que la acechan por su estrecho vínculo con la Secretaría de Salud. Sin embargo, la creación del Instituto Nacional de Salud Pública -y, dentro de este proceso, la modernización académica de la ESPM- se presenta hoy, con el paso del tiempo, como una epopeya de transformación institucional ampliamente reconocida alrededor del mundo. Como tal, ofrece una prueba estimulante de que la innovación es de hecho posible a pesar de la complejidad de los retos. Preservar la memoria histórica se vuelve, así, una fuente de optimismo sobre nuestra capacidad de mantener la marcha ascendente de la salud pública.

\section{Agradecimientos}

Los autores manifiestan su agradecimiento a las siguientes personas, quienes generosamente aceptaron ser entrevistadas como parte de la investigación para este ensayo: José Rodríguez-Domínguez, Francisco GarridoLatorre, Lizbeth López-Carrillo e Ignacio Villaseñor.

Declaración de conflicto de intereses. Los autores declararon no tener conflicto de intereses.

\section{Referencias}

I. Secretaría de Salud. Programa Nacional de Salud 200I-2006. La democratización de la salud en México. Hacia un sistema universal de salud. México, DF: Secretaría de Salud, 200I:33.

2. Frenk J, Sepúlveda J, Gómez-Dantés O, Knaul FM. Evidencebased health policy: three generations of reform in Mexico. Lancet 2003;362:|667-|67|

3. Decreto por el que se adiciona un párrafo al artículo $4^{\circ}$ de la Constitución Política de los Estados Unidos Mexicanos. Salud Publica Mex 1983;25(2):23|-232.

4. Ley General de Salud. Diario Oficial de la Federación, 7 de febrero 1984. 5. Secretaría de Salud (México) [consultado el 16 de enero de 2013]. Disponible en: http://es.wikipedia.org/wiki/Secretar\%C3\%ADa_de_ Salud_\%28M\%C3\%A9xico\%29

6. Soberón-Acevedo G, Martínez-Narváez G. La descentralización de los servicios de salud en México en la década de los ochenta. Salud Publica Mex 1996;38(5):37I-378.

7. Faculdade de Saúde Pública [consultado el 29 de enero de 2013] Disponible en: http://www.fsp.usp.br/site/paginas/mostrar//28

8. Rodrigues de Faria L. O Instituto de Higiene. Contribuica a história da ciencia a da administracan em saúde em São Paulo. Physis Rev Saúde Coletiva 1999;9(I):175-208.

9. Álvarez-Amézquita J, Bustamante M, López-Picasos A. La Escuela de Salud Pública de México. Setenta años de vida. Salud Publica Mex 1992;34(I):76-84

10. Rodríguez-Domínguez J. La Escuela de Salud Pública de México. Salud Publica Mex 1983;25(5):505-508.

II. Crónica de la Escuela de Salud Pública en el siglo XX. En:Valdespino, JL, Sepúlveda J, compiladores. Crónica de la Escuela de Salud Pública de México I 922 a 200I. Cuernavaca, México: Instituto Nacional de Salud Pública, 2002:17-23.

12. Moreno-Cueto E, Moguel J, Díaz de Sandi MA, García-Ugarte ME, Césarman-Vitis E. Sociología histórica de las instituciones de salud en México. México, DF: Instituto Mexicano del Seguro Social, 1982:6I-63. I3. Frenk J, Gómez-Dantés O. Para entender el sistema de salud de México. México, DF: Nostra Ediciones, 2008:25.
14. Molina G. Informe sobre las visitas a los países de América de profesores de las escuelas de salud pública que reciben estudiantes extranjeros. Boletín de la Organización Panamericana de la Salud 1954;37(I):6-10. 15. Escuela de Salud Pública de México. Modernización administrativa. México, D.F:: Instituto Nacional de Salud Pública, 1988.

16. Acuerdo por el que se delega en la Escuela de Salud Pública de México la facultad para el ejercicio administrativo de sus propios recursos. Salud Publica Mex 1982;XXIV(2):210-2II.

17. Soberón G. El cambio estructural en la salud. Salud Publica Mex 1987;29(2): 127-140.

18. Soberón G. El cambio estructural en la salud. III. La investigación y los recursos humanos como instrumentos del cambio. Salud Publica Mex 1987;29(2):155-165.

19. Consejo Nacional de Ciencia y Tecnología, Secretaría de Salud. Investigación de servicios de salud. Memoria del seminario llevado a cabo del 13 al 15 de julio de 1978 en Querétaro, México. México, DF: Conacyt, Secretaría de Salud, 1979.

20. Ortiz M. Devenir de la salud pública en México durante el siglo XX. Cuernavaca, Morelos, México: Instituto Nacional de Salud Pública, 2000: 45. 21. Horwitz A, Ferreira JR. Informe sobre la Escuela de Salud Pública de México. México, DF: Escuela de Salud Pública de México, 1985.

22. Bobadilla JL, Lozano R, Bobadilla C. Diseño curricular de programas de posgrado en salud: el caso de la Maestría en Epidemiología. Salud Publica Mex 1991;33:533-547.

23. Cenavece. Residencia en epidemiología [consultado el 20 de enero de 2013]. Disponible en: http://www.dgepi.salud.gob.mx/2010/plantilla/ intd_residencia.html

24. Centro de Investigaciones en Salud Poblacional.Antecedentes CISP [consultado el 22 de enero de 2013]. Disponible en: http://www.insp.mx/ centros/salud-poblacional/de-interes.html

25. Martuscelli J, Frenk J, Ruelas E, González-Block MA. La formación de recursos humanos para la salud en el contexto de la reordenación y reconstrucción de los servicios en el Valle de México. Rev Educacion Superior 1986;59:5-14.

26. Frenk J. Las oportunidades de la reconstrucción. Nexos 1985;VIII(I2). 27. Soberón G, Frenk J, Sepúlveda J.The health reform in Mexico: Before and after the earthquake.Am J Public Health 1986;76:673-680.

28. Ruelas-Barajas E. Nuevas alternativas de educación continua para directivos de servicios de salud. Salud Publica Mex 1987;29(5):442-447. 29. Ruelas-Barajas E, Durán L, García-Rojas M, Campero-Cuenca L, Hurtado A.Análisis de los problemas de los médicos directivos en diferentes organizaciones hospitalarias. I. Una metodología para la búsqueda e identificación de problemas.J Health Admon Educ 1990;8(2):340-358. 30. Ruelas-Barajas E, Durán L, García-Rojas M, Campero-Cuenca, Hurtado A .Análisis de los problemas de los médicos directivos en diferentes organizaciones hospitalarias. II. Formación académica y trayectoria laboral de los médicos directivos en México. J Heath Admon Educ 1990;8(3):479-509. 3I. Ruelas-Barajas E, Durán L, García-Rojas M, Campero-Cuenca, Hurtado A. Análisis de los problemas de los médicos directivos en diferentes organizaciones hospitalarias. III. Caracterización cuantitativa de los problemas percibidos por los médicos directivos en México.J Heath Admon Educ 1990;8(4): I46-162.

32. Ruelas-Barajas E, Durán L, García-Rojas M, Campero-Cuenca L, Hurtado A. Análisis de los problemas de los médicos directivos en diferentes organizaciones hospitalarias. IV: Caracterización cualitativa de los problemas percibidos por los médicos directivos en México.J Health Admon Educ 1991;9(I): 146-162.

33. Frenk J. La salud pública: campo del conocimiento y ámbito para la acción. Salud Publica Mex 1988;30(2):246-254.

34. Frenk J, Bobadilla JL, Sepúlveda J, Rosenthal J, Ruelas E. Un modelo conceptual para la investigación en salud pública. Bol Of Sanit Panam 1986; 101:477-492.

35. Cardozo-Brum M. Rediseño curricular de un programa de maestría en salud pública. Salud Publica Mex 1989;31(3):407-4I7. 